\title{
Publisher's Note: Robust quantum state transfer inspired by Dzyaloshinskii-Moriya interactions [Phys. Rev. A 95, 052332 (2017)]
}

X. Shi, H. Yuan, X. Mao, Y. Ma, and H. Q. Zhao

(Received 31 May 2017; published 6 June 2017)

DOI: 10.1103/PhysRevA.95.069901

This paper was published online on 16 May 2017 with incorrect statements in the Acknowledgments. The Acknowledgments should read as as "This work was supported in part by the National Natural Science Foundation of China through Grants No. 61601433, No. 11547105, and No. 11405171; the Chongqing Science and Technology Commission through Grant No. CSTC2013jcyj40001; the CAS Light of West China Program; the Anhui Provincial Natural Science Foundation through Grant No. 1608085QF139; and the China Postdoctoral Science Foundation through Grant No. 2016M600906." The Acknowledgments have been corrected as of 26 May 2017. The Acknowledgments are correct in the printed version of the journal. 